\title{
Oscillations superposées dans les cheminées d'équilibre à section constante ordinaires ou à étranglement
}

\section{Superposed oscillations in ordinary constant section or throttled surge tanks}

\author{
PAR L. ESCANDE \\ MEMBRE DE L'INSTITUT \\ LNGÉNIEUR I.E.T. \\ DIRECTEUR DE L'ÉCOLE NATIONALE SUPÉRIEURE D'ÉLECTROTTCHNIQUE ET D'HYDRAULIQUE DE TOULOUSE
}

\begin{abstract}
L'autent généralise le problème étudié dans un mémoire précédent (Mémoires et Travaux), $n^{\circ} 1-1954$, page 124 ) en tenant compte de l'étranglement et de la perte de charge. Cas des cheminées ordinaires. Ouverture et fermeture totales et particlles. Mise en évidence de l'instant d'intervention de la deuxième manxulve, pour lequel la superposition des oscillations provoque la plus forte montée ou descente et déterminalion de celles-ci. Comparaison de ces valeurs avec les déplacements extrềmes du plan d'ean provoqués par des manouvres isolées. Mèmes recherches dans le cas des cleeminées ḋ étranglement optimum pour la fermeture totale.

Abaques montrant que l'amplitude des oscillations superposées peut dépasser de beaucoup celles des oscillations engendrées par des manceuvres isolées.
\end{abstract}

\begin{abstract}
The author generalises the problem studied in a preceding memoire Mémoires et Travaux. $n^{\circ} 2-1954$, page 124) taking into account the throttle and the head losses.

Case of ordinary surge tanks. Total and parlial opening and shut down. Establishing the instant the 2nd manoupre intervenes to prodace, by superposilion of the oscillations, the greatest rise of fall-determination of the latter. Comparison of these values with lhe extreme displacements of the water surface cansed by isolaled manwenves. Same invesligations for surge lanks with the optimum throttle, for complete shat down.

Curves showing that the amplitude of the superposed oscillations can be very different from the oscillations caused by isolated manœuvres.
\end{abstract}

\section{I. - INTRODUCTION}

La plupart des cahiers des charges relatifs aux chambres d'équilibre prévoient que ces ouvrages doivent être établis en tenant compte des déplacements du plan d'eau provoqués par l'une et par l'autre des deux manouvres suivantes, considérée isolément :
1" Déclenchement intervenant lors d'une marche de régime permanent au débit maximum $Q_{0}$ et faisant passer le débit des turbines de $Q_{0}$ à 0 ;

2" Ouverture des turbines faisant passer le dé- 
bit de 0 à $Q_{0}$ ou de 0 à $\lambda Q_{0}$ avec $\lambda<1$, ou encore de $\lambda Q_{0}$ à 0 , cette prise de charge s'effectuant à partir d'un régime initial permanent.

Dans la majorité des cas, les cahiers des charges demandent de considérer les manœuvres comme instantanées, ce qui correspond à une hypothèse dans le sens de la sécurité, dans le cas des chambres ordinaires, mais peut conduire, au contraire, à une sous-estimation de l'amplitude des oscillations, vis-à-vis de celles qu'engendre une manœuvre d'une certaine durée, dans le cas des cheminées à étranglement, comme nous l'avons montré $\left({ }^{*}\right)$.

Dans la présente étude, nous désirons revenir sur une question importante que nous avons signalée à diverses reprises $\left(^{* *}\right)$ et qui concerne le danger que peut présenter la succession de deux manœuvres séparées par un intervalle de temps assez court pour que les oscillations engendrées par la première ne soient pas encore amorties lorsque se produit la seconde.

De telles circonstances risquent tout particulièrement de se rencontrer dans les centrales à débit important et long canal d'amenée pour lesquelles la période du système chambre d'équilibre canal d'amenée atteint et dépasse parfois un quart d'heure.

Dans le travail que résume cet article, nous avons considéré, tout d'abord, une cheminée d'équilibre ordinaire à section constante et envisagé la succession de deux manœuvres instantanées:

- soit ouverture de 0 à $Q_{0}$ suivie de fermeture de $\mathrm{Q}_{0}$ à 0 ,

- soit fermeture de $Q_{0}$ à 0 suivie d'ouverture de 0 à $Q_{0}$,
- soit fermeture de $Q_{0}$ à 0 suivie d'ouverture de 0 à $\lambda Q_{0}$ avec $\lambda<1$.

Nous avons mis en évidence, dans chaque cas, l'instant d'intervention de la seconde manouvre pour lequel la superposition des oscillations provoque la plus forte montée ou descente du plan d'eau et nous avons déterminé celle-ci, de même que la descente ou montée consécutive.

Nous avons comparé les valeurs ainsi obtenues aux déplacements extrêmes du plan d'eau habituellement considérés par les cahiers des charges, c'est-à-dire à la montée provoquée par une fermeture isolée de $Q_{0}$ à $O$ et à la descente engendrée par une ouverture isolée de 0 à $Q_{0}$ ou de 0 à $\lambda Q_{0}$.

Cette étude a été faite pour toutes les valeurs pratiquement intéressantes de la perte de charge $p_{0}$ dans le canal d'amenée.

Nous avons effectué ensuite la même recherche dans le cas des cheminées d'équilibre à étranglement, en nous limitant au cas de l'étranglement optimum pour la fermeture, c'est-à-dire celui pour lequel, lors d'un arrêt instantané du débit de $Q_{0}$ à 0 à partir d'un régime permanent, la surpression, dans le canal d'amenée, a la même valeur à l'instant initial, d'une part, et au moment où le plan d'eau atteint sa cote maximum, d'autre part.

Les résultats que nous avons obtenus montrent à quel point l'amplitude des oscillations superposées peut dépasser celle des oscillations engendrées par des manouvres isolées de fermeture ou d'ouverture.

Précisons que, dans toute cette étude, nous ne considérons que des manouvres instantanées.

\section{II. - NOTATIONS}

Les notations, à l'exception de $Q_{0}$, correspondent aux grandeurs relatives; les cotes $z$ du plan d'eau étant comptées à partir du niveau statjque, nous appelons :

$p_{0}$ la perte de charge dans le canal d'amenée pour le débit $Q_{0}$;

$r_{0}$ la perte de charge dans l'étranglement pour le débit $Q_{0}$;

$z_{u m}$ la cote maximum atteinte à la suite de l'ar-

(*) Comptes rendus de l'Académie des Sciences, C.R.A.S., t. 216, p. 193, 1943 et t. 230, p. 1932, 1950.

(“*) C.R.A.S., t. 214, p. 656, 1942 et t. 239, p. $5,1954$. rêt complet instantané du débit $Q_{0}$ (manœuvre unique);

$z_{u l}$ la cote la plus basse atleinte à la suite d'une ouverture instantanée de 0 à $Q_{0}$ (manœuvre unique);

$z_{\lambda u d}$ la cote la plus basse atteinte à la suite d'une ouverture instantanće de 0 à $\lambda Q_{0}$ avec $\lambda<1$ (manœuvre unique). Ies valeurs de $\lambda$ étudiées sont $0,25-0,50-0,75$; pour $\lambda=1$, on retrouve $z_{u d}$;

$z_{s m}$ cote maximum atteinte dans les conditions les plus dangereuses, par la succession d'une fermeture de $Q_{0}$ à 0 à une ouverture de 0 à $Q_{0}$; 
$z_{* t l}$ cote la plus hasse atteinte, au cours de cette même manœuvre, à la fin de la descente consécutive au passage du plan d'eau à la cote $z_{s m}$;

$z_{s i l}^{\prime}$ cote la plus basse atteinte, dans les conditions les plus dangereuses, par la succession d'une ouverture de 0 à $Q_{0}$ à une fermeture de $Q_{0}$ à 0 .

$z_{s, 1}^{\prime}$ cote maximum atteinte, au cours de cette même manœuvre, à la fin de la montée consécutive au passage du plan d'eau à la cote $z_{s i}^{\prime}$; $z_{\lambda s a c}^{\prime}$ cote la plus basse atteinte, dans les conditions les plus dangereuses, par la succession d'une ouverture de 0 à $\lambda Q_{0}$ à une fermeture de $Q_{0}$ à 0 , avec $\lambda=0,25-0,50$ $-0,75$. Pour $\lambda=-1$, on retrouve $z_{s d}^{\prime}$;

$z^{\prime} \lambda \operatorname{sm}$ cote maximum atteinte, au cours de celte même manœuvre, à la fin de la montée consécutive au passage du plan d'eau à la cote $z_{\lambda s d}^{\prime}$. Pour $\lambda=1$, on retrouve $z_{s m}^{\prime}$.

Les autres notations employées sont les notations classiques concernant les grandeurs relatives.

\section{III. - REMARQUE SUR LES METHODES DE CALCUL UTILISEES}

$1^{\circ}$ Pour plus de généralité, considérons une chambre à étranglement : il sera, en effet, facile de passer de là au cas d'une cheminée ordinaire en faisant $r=0$ dans les équations.

Considérons tout d'abord une ouverture de 0 à $Q_{0}$ pour laquelle la méthode graphique classique de Calame et Gaden permet de construire sans difficultés la courbe $(z, v)$.

Soit $z_{1}, v_{1}$ les coordonnées au point de fonctionnement, sur cette courbe, au moment où se produit le déclenchement qui fait passer instarilanément le débit des turbines de $Q_{0}$ à 0 .

Dans la zone intéressante pour notre problème, le point $\left(z_{1}, w_{1}\right)$ correspond à des valeurs positives de $v_{1}$ et $w_{1}$, de telle sorte qu'après le déclenchement, l'équation des oscillations s'écrit:

$$
v \frac{d v}{d z}+z+p+r=0
$$

avec :

$$
\begin{gathered}
u=0 ; \quad w=v \\
p=p_{0} w^{2}=p_{0} v^{2}, \quad r=r_{0} v^{2}, \quad p+r=\left(p_{0}+r_{0}\right) v^{2}
\end{gathered}
$$

on en déduit :

$$
\frac{d v}{d z}=-\frac{z+p+r}{v}
$$

et :

$$
\begin{array}{r}
\frac{d^{2} v}{d z^{2}}=-\frac{1}{v^{3}}\left[v^{2}+(z+p+r)(z-p-r)\right] \\
=--\frac{1}{v^{3}}\left[v^{2}+z^{2}-(p+r)^{2}\right]
\end{array}
$$

L'expression du rayon de courbure est alors :

$$
\rho=\frac{\left[v^{2}+(z+p+r)^{2}\right]^{3 / 2}}{\left|v^{2}+z^{2}-(p+r)^{2}\right|}
$$

On a ainsi la pente de la tangente et le rayon de courbure initiaux au point $\left(z_{1}, n_{j}\right)$ considéré comme point de départ de l'oscillation provoquée par la manœuvre de fermeture :

$$
\begin{gathered}
\left(\frac{d v}{d z}\right)_{3}=-\frac{z_{1}+p_{1}+v_{1}^{\prime}}{v_{1}^{\prime}} \\
q_{1}=\frac{\left[v_{1}^{\prime}+\left(z_{1}+p_{1}+r_{1}^{\prime}\right)^{2}\right]^{3 / 2}}{\left|v_{1}^{\prime}+z_{1}^{2}-\left(p_{1}+r_{1}^{\prime}\right)^{2}\right|}
\end{gathered}
$$

avec :

$$
v_{1}^{\prime}=v_{1}+1 \quad r_{1}^{\prime}=r_{0} v_{1}^{\prime}{ }^{2}=r_{0}\left(v_{1}+1\right)^{2}
$$

L'applicalion de la construction graphique classique permet alors, par le procédé des normales, la détermination de la courbe $(z, v)$ et du maximum de celle-ci correspondant an moment oì $b=0$.

2" Dans le cas d'une chambre d'épuilibre ordinaire, la méthode se simplifie.

On a pour la deuxieme oscillation :

$$
\begin{gathered}
v \frac{d v}{d z}+z+p=0 \\
\frac{d v}{d z}=-\frac{z+p}{v} \text { avec } p=p_{0} v^{2} \\
\frac{d^{2} v}{d z^{2}}=-\frac{1}{v^{3}}\left[v^{2}+z^{2}-p^{2}\right] \\
\left(\frac{d v}{d z}\right)_{1}=-\frac{z_{1}+p_{1}+v_{1}^{\prime}}{v_{1}^{\prime}} \\
\rho_{1}=\frac{\left[v_{1}^{2}+\left(z_{1}+p_{1}\right)^{2}\right]^{3 / 2}}{\left|v_{1}^{2}+z_{1}^{2}-p_{1}^{2}\right|}, \text { avec } v_{1}^{\prime}=v_{1}+1
\end{gathered}
$$

$3^{\circ}$ Revenons au cas général de la chambre à étranglement, mais considérons maintenant, en 
premier lieu, une fermeture instantanée de $Q_{0}$ à 0 , pour laquelle les méthodes classiques permettent de déterminer la courbe $(z, v)$ et soit $z_{1}, v_{1}$ le point de fonctionnement pour lequel se produit l'ouverture instantanée de 0 à $Q_{0}$. Dans la zone intéressante pour notre problème, $v_{1}$ et $w_{1}$ sont négatifs, de telle sorte qu'au début de la nouvelle oscillation les équations s'écrivent :

$\frac{d v}{d z}=-\frac{z-p-r}{v}$ avec $p=p_{0}(v+1)^{2}, \quad r=r_{0} v^{2}$

$\frac{d^{2} v}{d z^{2}}=-\frac{1}{v^{3}}\left\{v^{2}+(z-p-r)\left[z-p+r+2 p_{0} v(v+1)\right]\right\}$

$?=\frac{\left[v^{2}+(z-p-r)^{2}\right]^{3 / 2}}{\left|v^{2}+(z-p-r)\left[z-p+r+2 p_{0} v(v+1)\right]\right|}$

L'ouverture instantanée fait passer instantar nément $v$ de $v_{1}$ à la valeur initiale :

$$
v_{1}^{\prime}=v_{1}-1
$$

à laquelle correspond la valeur initiale de $r$ :

$$
r_{1}^{\prime}=r_{0} v_{1}^{\prime}{ }^{2}=r_{0}\left(v_{1}-1\right)^{2}
$$

on en déduit :

$$
\frac{d v_{1}}{d z_{1}}=-\frac{z_{1}-p_{1}-r_{1}^{\prime}}{v_{1}^{\prime}}
$$

$P_{1}=\frac{\left[v_{1}^{\prime}+\left(z_{1}-p_{1}-r_{1}^{\prime}\right)^{2}\right]^{3 / 2}}{\left|v_{1}^{\prime}{ }^{2}+\left(z_{1}-p_{1}-r_{1}^{\prime}\right)\left[z_{1}-p_{1}+r_{1}^{\prime}+2 p_{0} v_{1}^{\prime}\left(v_{1}^{\prime}+1\right)\right]\right|}$

On a ainsi le point de départ de la courbe $(z, v)$ relative à la deuxième oscillation que l'on étudie jusqu'à ce que soit atteinte la cote la plus basse du plan d'eau en tenant compte du changement de signe de $w$ qui se produit au cours de la descente et de sa répercussion sur la construction graphique;

$4^{\circ}$ On passe facilement de ce cas à celui d'une chambre d'équilibre ordinaire, en faisant dans toutes les équations précédentes $r=0$ comme nous l'avons dit;

$5^{\circ}$ Le cas où une ouverture de 0 à $\lambda Q_{0}$ succède à une fermeture de $Q_{0} \dot{a} 0$ se traite de facon analogue, aussi bien pour la chambre d'équilibre à étranglement que pour la chambre d'équilibre ordinaire.

\section{IV. - APPLICATION A UNE CHAMBRE D'EQUILIBRE ORDINAIRE : \\ Cas d'une fermeture de $Q_{0}$ à 0 succédant à une ouverture de 0 à $Q_{v}$}

Nous supposons qu'à l'instant initial, tout étant au repos, le débit des turbines passe instantanément de 0 à $Q_{0}$ et nous déterminons, au moyen de la méthode graphique, la courbe $(z, v)$ donnant, en grandeurs relatives, la vitesse $v$ du plan d'eau dans la chambre d'équilibre en fonction de la cote $z$ au-dessus du nivean statique.

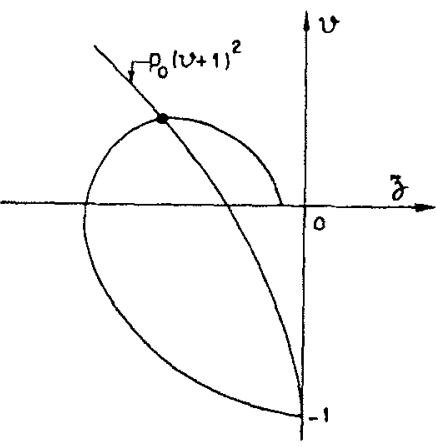

Frg. 1

Soudain, un déclenchement fait passer instantanément le débit des turbines de $Q_{0}$ à 0 . L'oscillation qui se greffe ainsi sur la première atteint une amplitude variable selon l'instant du déclenchement. Nous cherchons, en faisant varier celui-ci, les conditions de production de la mon- tée maximum $z_{s m}$ dı plan d'eau. Nous trouvons que celle-ci est réalisée lor'sque l'instant du déclenchement correspond au maximum de $v$ dans

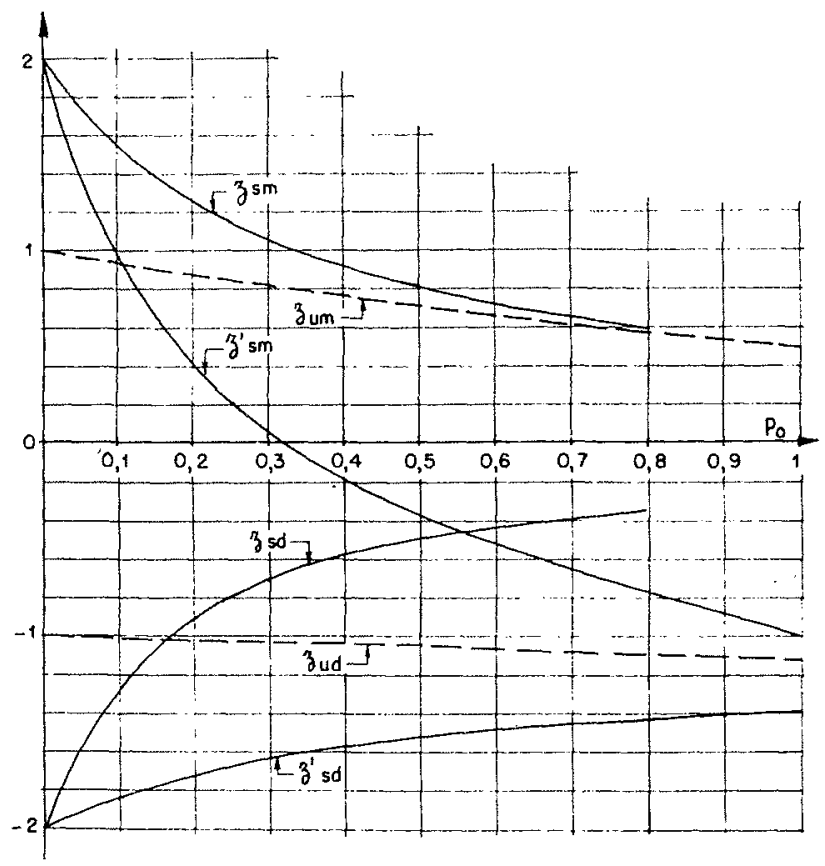

Fig. 2 


\section{TABLEAU I}

\begin{tabular}{|c|c|c|c|c|c|c|c|}
\hline$p_{0} \ldots \ldots$ & 0 & 0,1 & 0,2 & 0,4 & 0,6 & 0,8 & 1 \\
\hline$z_{u m} \ldots \ldots$ & 1 & 0,935 & 0,875 & 0,755 & 0,65 & 0,555 & \\
\hline$z_{u d} \ldots \ldots$ & -1 & $-1,012$ & $-1,025$ & $-1,05$ & $-1,075$ & $-1,103$ & \\
\hline$z_{s m} \ldots \ldots$ & 2 & 1,555 & 1,25 & 0,91 & 0,72 & 0,579 & \\
\hline$k_{m} \ldots \ldots$ & 2 & 1,662 & 1,43 & 1,205 & 1,107 & 1,042 & \\
\hline$z_{s d} \ldots \ldots$ & -2 & $-1,295$ & $-0,93$ & $-0,585$ & $-0,45$ & $-0,347$ & \\
\hline$k_{d} \ldots \ldots$ & 2 & 1,28 & 0,907 & 0,557 & 0,418 & 0,314 & \\
\hline$z_{s}^{\prime} \ldots \ldots$ & -2 & $-1,848$ & $-1,719$ & $-1,580$ & $-1,493$ & $-1,437$ & $-1,397$ \\
\hline$k_{d}^{\prime} \ldots \ldots$ & 2 & 1,828 & 1,677 & 1,503 & 1,389 & 1,302 & 1,215 \\
\hline$z_{s m}^{\prime} \ldots \ldots$ & 2 & 0,962 & 0,402 & $-0,192$ & 0,55 & $-0,787$ & -1 \\
\hline$k_{m}^{\prime} \cdots$ & 2 & 1,029 & 0,46 & $-0,254$ & $-0,846$ & $-1,420$ & $-2,10$ \\
\hline
\end{tabular}

l'oscillation initiale provoquée par l'ouverture : on vérifie facilement que ce maximum de $v$ se situe au point d'intersection de la courbe $(z, v)$ et de la courbe intermédiaire $p=p_{0}(1+v)^{2}$ utilisée pour la construction graphique (fig. 1).

Nous déterminons ainsi $z_{s m}$ pour différentes valeurs de $p_{0}$ et nous posons :

$$
z_{s m n}=z_{u m} \times k_{m}
$$

$z_{\text {tm }}$ désignant la cote maximum atteinte à la suite d'un arrêt complet instantané, en l'absence d'oscillations préexjstantes. Le tableau I et la figure 2 donnent $z_{s m}, z_{u m}$ et $k_{m}$ en fonction de $p_{0}$ : quand $p_{0}$ croît de 0 à $0,8, k_{m}$ diminue progressivement de 2 à 1,04; l'amplification des oscillations est d'autant plus grande que la perte de charge est plus faible.

Nous déterminons également la descente maximum consécutive du plan d'eau $z_{s d}$ et nous la comparons à celle $z_{u d}$ que provoque une ouverture instantanée de 0 à $Q_{0}$ à partir du repos, en posant :

$$
z_{s l}=z_{u d} \times k_{d}
$$

Comme on le voit sur le tableau I et la figure 2, c'est seulement pour de très faibles pertes de charge $\left(p_{0}\right.$ inférieur à 0,17$)$ que l'on a $k_{d}>1$.

\section{V. - APPLICATION A UNE CHAMBRE D'EQUILIBRE ORDINAIRE : \\ Cas d'une ouverture de 0 à $Q_{0}$ succédant à une fermeture de $Q_{0}$ à 0}

C'est le problème inverse du précédent : apròs un déclenchement intéressant le débit maximum, on passe à la pleine ouverture des turbines, les manceuves étant toujours supposées instantanées.

L'ouverture qui produit la plus forte descente $z_{\text {sd }}^{\prime}$ du plan d'eau correspond à un point $\left(z_{1}, v_{1}\right)$ confondu avec le minimum de la courbe $(z, v)$, relative à la fermeture initiale, point d'intersection de cette courbe et de la courbe $p(v)$ de la perte de charge (fig. 3). Nous posons:

$$
z_{s d}^{\prime}=z_{u d} \times k_{d}^{\prime}
$$

Les tableaux I et II et les figures 2 et 4 donnent $z_{s d}^{\prime}, z_{u d}, k_{d}^{\prime}$ en fonction de $p_{0}$.

$k_{q}^{\prime}$ décroît de 2 a 1,215 quand $p_{0}$ passe de 0 à 1 .

Nous comparons également à $z_{u m}$ la monlée maximum $z_{s m}^{\prime}$ consécutive à la descente $z_{s d}^{\prime}$, en posant :

$$
z_{s m}^{\prime}=z_{u m} \times k_{m}^{\prime}
$$

On voit (tableaux I et II el figures 2 el 4) que, saul pour $p_{0}<0,10$, $z^{\prime}{ }_{s m}$ est inférieur à $z_{u m}$.

Vis à vis des amplitudes calculées à partir de mancuves isolées de fermcture ou d'ouverture,

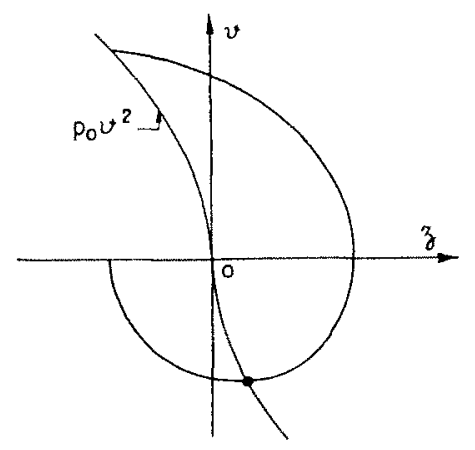

FIG. 3

la succession de ces deux mancuvres peut conduire, dans tous les cas, a une augmentation des amplitudes de la première oscillation consćcu- 
TABLEAU II

\begin{tabular}{|c|c|c|c|c|c|c|c|c|}
\hline & $p_{0} \ldots \ldots \ldots$ & 0 & 0,1 & 0,2 & 0,4 & 0,6 & 0,8 & 1 \\
\hline & $z_{u m} \ldots \ldots \ldots !$ & 1 & 0,935 & 0,875 & 0,755 & 0,65 & 0,555 & 0,475 \\
\hline \multirow{5}{*}{ 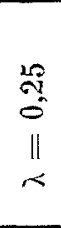 } & $z_{0,25 s d^{\prime}}^{\prime} \ldots !$ & $-1,25$ & $-1,097$ & $-0,97$ & $1-0,800$ & $-0,697$ & $-0,614$ & $-0,561$ \\
\hline & $z_{0,20} u d \cdots$ & $-0,25$ & $-0,252$ & $-0,253$ & $-0,254$ & $-0,255$ & $-0,257$ & $-0,259$ \\
\hline & $k_{0.25 a}^{\prime} \cdots$ & 5 & 4,36 & 3,84 & 3,15 & 2,74 & 2,39 & 2,16 \\
\hline & $z_{0,25 s m}^{\prime} \ldots$ & 1,25 & 0,885 & 0,654 & 0,400 & 0,249 & 0,158 & 0,09 \\
\hline & $k_{0.25 m}^{\prime} \cdots$ & 1,25 & 0,945 & 0,747 & 0,530 & 0,383 & 0,285 & 0,189 \\
\hline \multirow{5}{*}{$\begin{array}{l}\text { 念 } \\
0 \\
11 \\
2\end{array}$} & $z_{0.58 d}^{\prime} \ldots .1$ & $-1,5$ & $-1,35$ & $-1,227$ & $-1,062$ & $-0,963$ & $-0,882$ & $-0,836$ \\
\hline & $z_{0,5 u d^{*}} \ldots$ & $-0,5$ & $-0,502$ & $-0,506$ & $-0,51$ & $-0,516$ & $-0,525$ & $-0,533$ \\
\hline & $k_{0.5 d}^{\prime} \cdots$ & 3 & 2,69 & 2,43 & 2,08 & 1,87 & 1,68 & 1,57 \\
\hline & $z_{0, a-u a^{0}} \ldots$ & 1,5 & 0,953 & 0,623 & 0,254 & 0,058 & $-0,081$ & $-0,190$ \\
\hline & $k_{0.5 m}^{\prime} \ldots$ & $1, \overline{5}$ & 1,02 & $0, \overline{712}$ & 0,336 & 0,089 & $-0,146$ & $--0,4$ \\
\hline \multirow{5}{*}{ 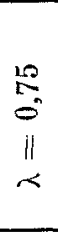 } & $z_{0,758 d}^{\prime} \cdots \cdot$ & $-1,75$ & $-1,603$ & $-1,48$ & $-1,328$ & $-1,230$ & $-1,152$ & $-1,106$ \\
\hline & $z_{0,75 u d} \ldots 1$ & $-0,75$ & $1-0,755$ & $-0,762$ & $1-0,78$ & $-0,79$ & $-0,803$ & $-0,82$ \\
\hline & $k_{0,75 a}^{\prime} \ldots$ & 2,33 & 2,12 & 1,94 & $1 \quad 1,7$ & 1,558 & 1,436 & 1,348 \\
\hline & $z_{0.758 m}^{\prime} \ldots !$ & 1,75 & 0,98 & 0,547 & 0,06 & $-0,216$ & $-0,404$ & $-0,556$ \\
\hline & $k_{0.75 \mathrm{~m}}^{\prime} \cdots$ & 1,75 & 1,048 & 0,625 & 0,079 & $\ldots-0,332$ & $!-0,728$ & $-1,17$ \\
\hline \multirow{5}{*}{$\vec{I}_{\underline{\sim}}$} & $z_{s d}^{\prime} \ldots \ldots \mid$ & -2 & $1-1,848$ & $-1,719$ & $1-1,580$ & $-1,493$ & $-1,437$ & $-1,397$ \\
\hline & $z_{u d d} \ldots \ldots$ & -1 & $-1,012$ & $-1,025$ & $-1,05$ & $-1,075$ & $-1,103$ & $-1,15$ \\
\hline & $k_{d}^{\prime} \ldots \ldots$ & 2 & 1,828 & 1,677 & 1,503 & 1,389 & $1 \quad 1,302$ & 1,215 \\
\hline & $z_{s m}^{\prime} \ldots \ldots 1$ & 2 & 0,962 & 0,402 & $-0,192$ & $-0,55$ & $-0,787$ &.-1 \\
\hline & $k_{n}^{\prime} \ldots \ldots$ & 2 & 1,029 & 0,46 & $-0,254$ & $-0,846$ & $-1,420$ & $-2,1$ \\
\hline
\end{tabular}

live à la deuxième manouvre: cette augmentation est d'autant plus forte que $p_{0}$ est petit. Pour l'oscillation en sens inverse successive, il n'y a majoration vis-à-vis du premier maximum atteint dans le cas d'une manœuvre isolće que pour des valeurs très faibles de $p_{0}$.

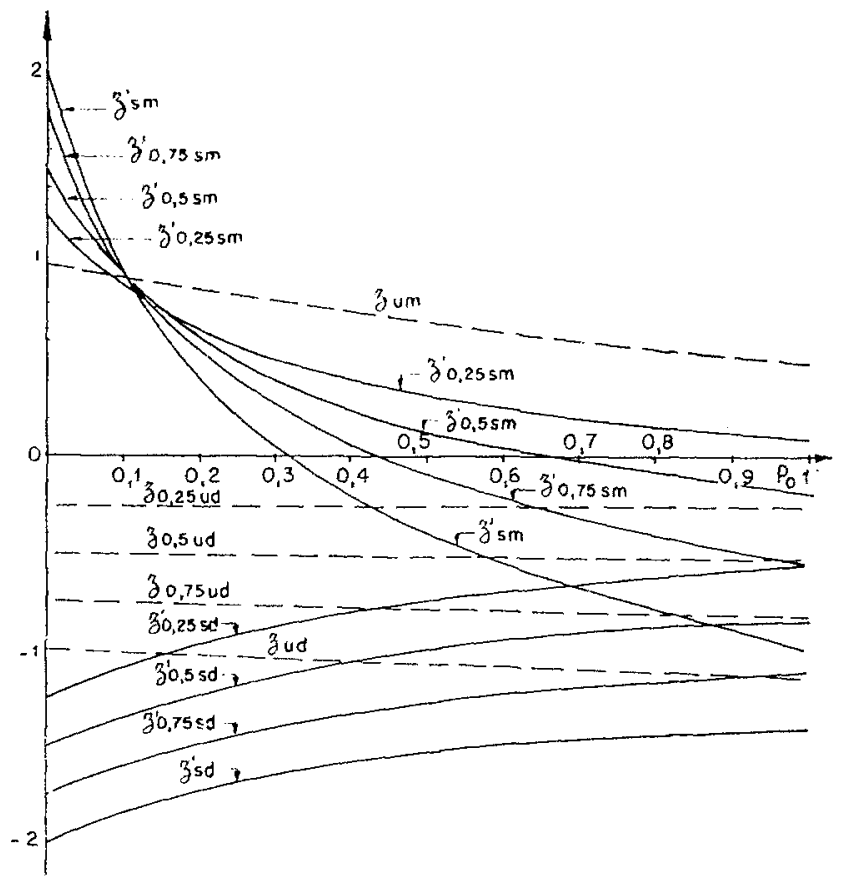

FIG. 4 


\section{VI. - APPLICATION A UNE CHAMBRE D'ÉQUILIBRE ORDINAIRE :}

\section{Cas d'une ouverture partielle succédant à une fermeture de $Q_{0}$ à 0}

Dans ce qui précède, nous avons étudié le cas où une ouverture de 0 à $Q_{0}$ succède à une fermeture de $Q_{0}$ à 0 alors que les oscillations engendrées par cet arrêt brusque du débit ne sont pas. encore amorties. En fait, si le déclenchement à pleine charge ne peut être évité, l'exploitant reste maître de limiter, dans une certaine mesure, les prises de charge d'une usine : pour cette raison, les chambres d'équilibre ne sont prévues, le plus souvent, que pour des manœuvres d'ouverture intéressant seulement une fraction du débit total. Nous avons envisagé ce cas et nous résumons les résultats que nous avons obtenus lorsqu'une ouverture de 0 à $\lambda Q_{0}$ (avec $\lambda$ égal à $0,75-0,50$ $-0,25)$ succède à une fermeture de $Q_{0}$ à 0 , les manouvres étant toujours considérées comme instantanées.

Nous avons constaté, comme précédemment pour $\lambda=1$, que la plus forte descente $z_{\lambda s ? \text { est }}^{\prime}$ obtenue quand l'ouverture correspond à un point $\left(z_{1}, v_{1}\right)$ confondu avec le minimum de la courbe $(z, v)$ relative à la fermeture initiale, point d'intersection de cette courbe et de la courbe $p(v)$ de la perte de charge. Nous avons ainsi déterminé $z_{\lambda s d}$ pour diverses valeurs de la perte de charge $p_{0}$ dans le canal d'amenée et nous l'avons comparé, en posant :

$$
z_{\lambda s d}^{\prime}=z_{\lambda u d} \times k_{\lambda d}^{\prime}
$$

à la cote minimum $z_{\lambda u d}$ atteinte par le plan d'eau dans la chambre d'équilibre à la suite d'une manœuvre isolée d'ouverture partielle, de 0 à $\lambda Q_{0}$, intervenant à partir du repos.

Nous avons comparé également le maximum de la montée du niveau $z_{\lambda s m}^{\prime}$, succédant à la descente $z_{\lambda s d}^{\prime}$, à la montée maximum $z_{z m m}$, provoquée par le déclenchement initial (arrèt instantané de $Q_{0}$ à 0 à partir du régime permanent de débit $\left.Q_{0}\right)$, en posant :

$$
z^{\prime}{ }_{\lambda s m}=z_{u m} \times k_{\lambda m}^{\prime}
$$

Les résultats obtenus sont fournis par le tableau II et la figure 4.

On voit que, pour toutes les valeurs de $\lambda$ et dans les limites de pertes de charge étudiées $\left(p_{0}<1\right), z_{\lambda s d}^{\prime}$ est toujours supérieur, en valeur absolue, à $z_{\lambda u d}$, la majoration étant d'autant plus grande que $\lambda$ et $p_{0}$ sont plus faibles.

Par contre, sauf pour $p_{0}<0,12, z_{\lambda \mathrm{s} m}^{\prime}$ est toujours inférieur à $z_{u m m}$, l'écart croissant avec la valeur de $\lambda$.

Dans le cas des chambres d'équilibre calculées pour des manœuvres d'ouverture partielles, une prise de charge, intervenant à la suite d'uu déclenchement à plein débit, alors que les oscillations du plan d'eau ne sont pas calmées, peut provoquer une descente du plan d'eau très supérieure à celle que donne une manouvre isolée d'ouverture à partir du repos: ainsi pour $\lambda=0,25$, le coefficient de majoration $k_{0,25 d}^{\prime}$ varie de 5 à 2,16 quand $p_{0}$ passe de 0 à 1 .

\section{VII. - CAS D'UNE CHAMBRE D'ÉQUILIBRE A ÉTRANGLEMENT}

Dans ce qui précède, nous avons étudié le problème de la superposition des oscillations dues à deux manœuvres instantanées inverses consécutives dans une chambre d'équilibre ordinaire.

Etant donné l'importance pratique des chambres d'équilibre à étranglement, nous avons effectué la même étude pour de tels ouvrages supposés équipés avec l'étranglement optimum $r_{0}$ relatif à une fermeture totale instantanée du débit maximum $Q_{0}$, pour chaque raleur de $p_{0}$.

Les manouvres successives considérées et les notations adoptées demeurant les mêmes que précédemment, nous résumons dans le tableau III et la figure 5 les résultats que nous avons obtenus pour les chambres d'équilibre à étranglement.

De façon générale, on observe que les superpositions d'oscillations, tout en pouvant provoquer, dans certains cas, des amplitudes dangereuses, produisent toutefois une amplification de

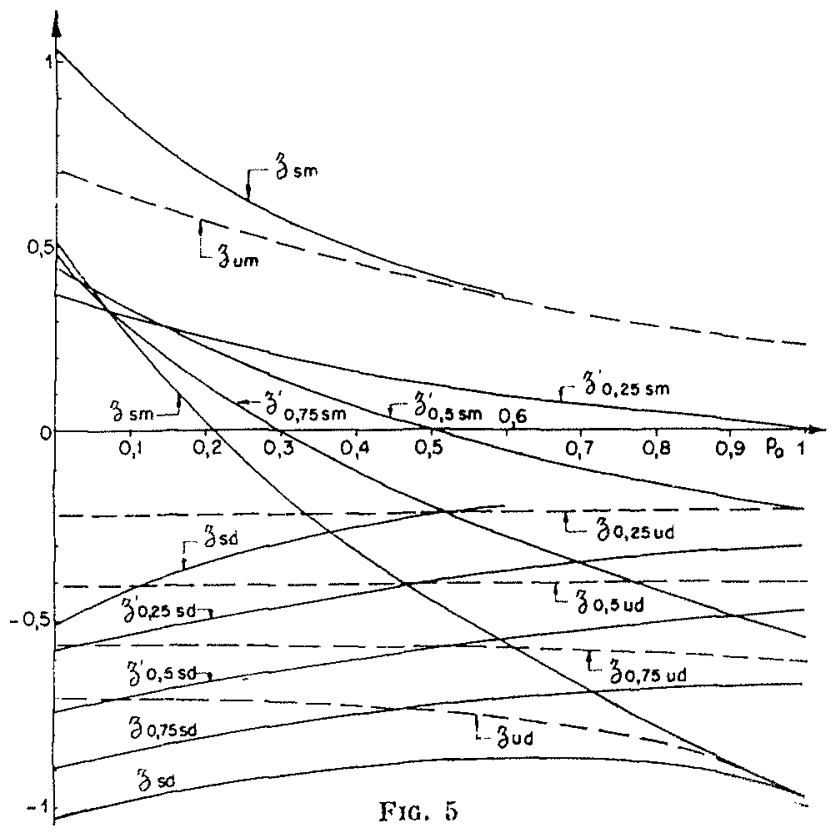


TABLEAU III

\begin{tabular}{|c|c|c|c|c|c|c|c|}
\hline$p_{0} \ldots \ldots \ldots \ldots$ & 0 & 0,1 & 0,2 & 0,4 & 0,6 & 0,8 & 1 \\
\hline$r_{0} \ldots \ldots \ldots \ldots$ & 0,707 & 0,738 & 0,775 & 0,850 & 0,960 & 1,08 & 1,23 \\
\hline$z_{u m} \ldots \ldots \ldots \ldots$ & 0,707 & 0,64 & 0,57 & 0,455 & 0,357 & 0,28 & 0,23 \\
\hline$z_{i m} \ldots \ldots \ldots \ldots$ & 1,028 & 0,830 & 0,687 & 0,49 & 0,365 & & 1 \\
\hline$k_{m} \ldots \ldots \ldots \ldots$ & 1,455 & 1,297 & 1,205 & 1,076 & 1,021 & & 1 \\
\hline$z_{u d} \ldots \ldots \ldots \ldots \ldots$ & $-0,707$ & $-0,713$ & $1-0,72$ & $-0,74$ & $-0,775$ & $-0,84$ & $1-1$ \\
\hline$z_{s i d} \ldots \ldots \ldots \ldots$ & $-0,512$ & $-0,420$ & $-0,354$ & $-0,262$ & $-0,204$ & & 1 \\
\hline$k_{a} \ldots \ldots \ldots \ldots$ & 0,725 & 0,589 & 0,491 & 0,354 & 0,263 & & 1 \\
\hline$z_{0,25 s d^{\prime}} \ldots$ & $-0,577$ & & $1-0,507$ & $1-0,436$ & $-0,38$ & $-0,34$ & $1-0,316$ \\
\hline$z_{0.25 n d} \ldots$. & $-0,224$ & & $-0,224$ & $-0,225$ & $-0,22$ & $-0,2175$ & $-0,216$ \\
\hline$k_{0.25 d}^{\prime} \cdots !$ & 2,58 & & 2,26 & 1,96 & 1,728 & 1,563 & 1,463 \\
\hline$z_{0.25 \mathrm{~m}}^{\prime} \cdots 1$ & 0,372 & & 0,252 & 0,161 & 0,092 & 0,05 & 0,003 \\
\hline$k_{0,25 m}^{\prime} \ldots$ & 0,526 & & 0,442 & 0,354 & 0,258 & 0,1786 & 0,013 \\
\hline$z_{0,5 s a}^{\prime} \ldots$ & $-0,740$ & & $-0,670$ & $-0,612$ & $1-0,553$ & $-0,523$ & $-0,487$ \\
\hline$z_{0,5 s m^{\prime}}^{\prime} \ldots$ & $-0,41$ & & $-0,41$ & $-0,41$ & $1-0,41$ & $-0,412$ & $-0,412$ \\
\hline$k_{0,5}^{\prime} d \ldots$ & 1,803 & & 1,633 & 1,493 & 1,35 & 1,27 & 1,182 \\
\hline$z_{0,5 s m^{\prime}} \ldots \mid$ & 0,44 & & 0,222 & 0,062 & $-0,056$ & $-0,15$ & $-0,222$ \\
\hline$k_{0.5 m}^{\prime} \ldots$. & 0,622 & & $1 \quad 0,39$ & $0, \mathbf{1 3 6}$ & $-0,157$ & $-0,536$ & $-0,965$ \\
\hline$z_{0.75 s d}^{\prime} \cdots \cdot 1$ & $-0,891$ & & $1-0,816$ & $-0,759$ & $-0,72$ & $-0,695$ & $-0,689$ \\
\hline$z_{0 . \pi n u d} \ldots$ & $-0,567$ & & $-0,57$ & $-0,578$ & $-0,585$ & $-0,604$ & $--0,626$ \\
\hline$k_{0 . \tau \bar{J} d}^{\prime} \cdots !$ & 1,57 & & 1,43 & 1,312 & 1,23 & 1,15 & 1,1 \\
\hline$z_{0 . \tau 5 \mathrm{sm}}^{\prime} \ldots$ & 0,480 & & 0,123 & $-0,110$ & $-0,288$ & $-0,435$ & $1-0,56$ \\
\hline$K_{0.75 m}^{\prime} \cdots$ & 0,68 & & 0,216 & $-0,242$ & $-0,806$ & $-1,552$ & $-2,43$ \\
\hline$z_{s d}^{\prime} \ldots \ldots$ & $-1,028$ & & $1-0,953$ & $-0,902$ & $-0,881$ & $-0,9$ & -1 \\
\hline$z_{u l d} \ldots \ldots !$ & $-0,707$ & & $-0,72$ & $-0,74$ & $--0,775$ & $-0,84$ & -1 \\
\hline$k_{d l}^{\prime} \ldots \ldots$ & 1,455 & 1 & 1,32 & 1,22 & 1,137 & 1,072 & 1 \\
\hline$z_{s m}^{\prime} \ldots .$. & 0,512 & & 0,027 & $-0,317$ & $-0,581$ & $-0,798$ & $1-1$ \\
\hline$k_{n}^{\prime} \ldots$ & 0,725 & & 0,047 & $-0,696$ & $--1,625$ & $-2,85$ & $\mid-4,35$ \\
\hline
\end{tabular}

celles-ci moindre que dans le cas des chambres d'équilibre ordinaires et cela d'autant plus que $p_{0}$ et par suite $r_{0}$ sont plus grands.

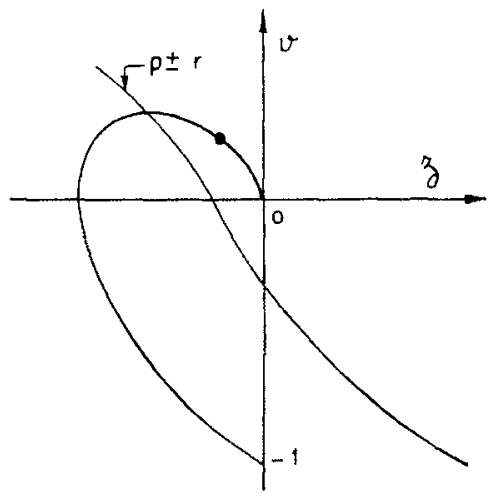

Fig. 6

On peut observer également que l'instant dintervention de la deuxième manœuvre, pour lequel l'oscillation superposée atteint le maximum d'amplitude, ne correspond pas exactement à l'in- tersection de la courbe $(v, z)$ et de la courbe donnant $p+r$ en fonction de $v$, c'est-à-dire, au maximum de la valeur absolue de $v$, mais à un

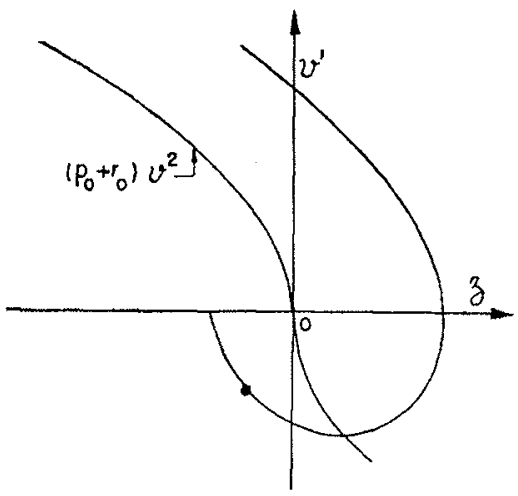

Fig. 7

instant légèrement postérieur, comme le montrent la figure 6 (fermeture $100 \%$ succédant à une ouverture $100 \%$ ) et la figure 7 (ouverture $100 \%$ succédant à une fermeture $100 \%$ ). 


\section{DISCUSSION}

Président: M. Gibrat

\section{Bouvard fait l'exposé ci-après :}

\section{OSCILLATIONS SUPERPOSÉES \\ DANS UNE CHEMINÉE A ÉTRANGLEMENT}

\section{ETUde PAR LES PROCÉDÉS RÉTROGRADES}

M. Molbert et moi-même avons étudié le problème des reprises en charge après déclenchement d'une usine hydraulique alimentée par une galerie en charge, avec cheminée d'équilibre, à propos du calcul des oseillations en masse dans la galerie de la chute Isère/Arc. Le résumé en est donné rapidement dans l'article que nous avons publié dans la Houille Blanche, $\mathrm{n}^{\circ} 2$, 1953, page 275.

On considere une courbe d'oscillation a la fermeture, après déclenchement. et une courbe d'oscillation lors d'une ouverture instantanée, construite en partant du fond de la cheminée (courbe rétrograde) :

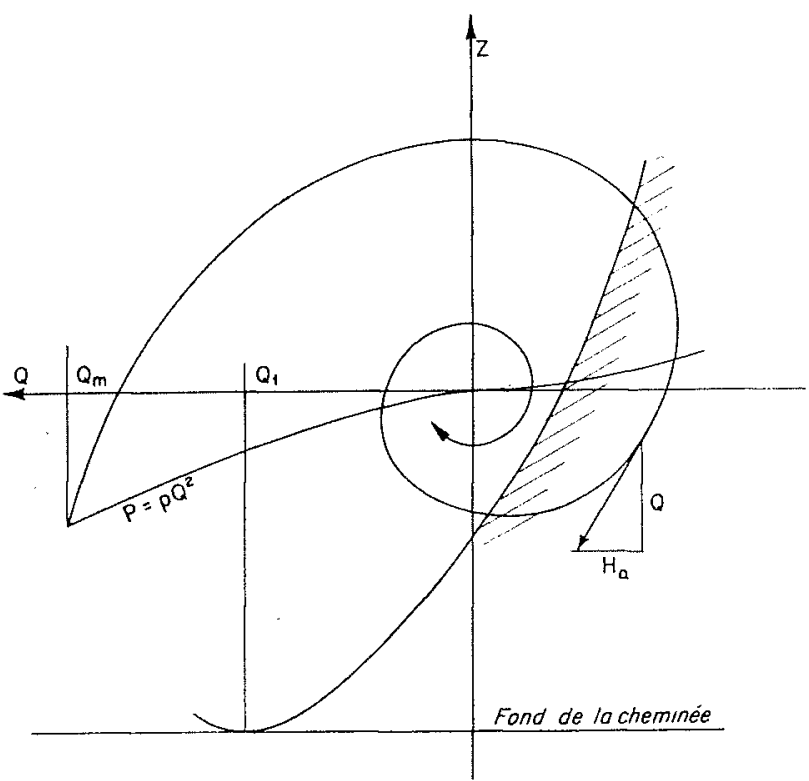

FIG. 1

- Si elles se coupent: les manouvres ne peuvent se faire qu'à gauche de la zone d'intersection;

- Si elles ne se coupent pas : les manceuvres peuvent se faire sans découvrir le fond de la cheminée.

On peut done remarquer :

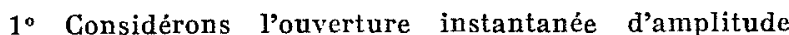
maximum qu'on peut effectuer à partir d'un débit nul dans la galerie. La courbe d'oscillation correspondante passe par l'origine des axes (coordonnées : $Z$, niveau dans la cheminée; $Q$, débit dans la galerie). La construction, indiquée précédemment, permet de remarquer qu'il faut en réalité prohiber cette manœuvre après un déclenchement pendant plus de la moitié de la durée des oscillations consécutives à une ouverture (plus de la moitié à cause de la concavité de la courbe d'ouverture, qui laisse dans la zone prohibée plus de la moitié de la courbe de fermeture). $2^{\circ}$ Le point le plus défavorable, pour ouvrir instantanément d'une quantité donnée, après un déclenchement, est celui où la courbe rétrograde est tangente da la courbe de fermeture.

On peut déterminer aisément, en un point représentant le mouvement après déclenchement, quelle est la manœuvre d'ouverture limite, avec un étranglement créant une perte de charge quelconque, donnée. Pour que les courbes soient tangentes, il faut en effet:

$$
\frac{Q_{0}+Q_{1}}{Q_{0}}=\frac{H_{a}+\Delta H_{a}}{H_{a}}
$$

Ha représente la hauteur accélératrice au point considéré, dans l'oscillation qui suit la fermeture,

$Q_{0}$ le débit au inême point

$Q_{1}$ le débit d'ouverture supposée instantanée,

$\Delta \mathrm{H}_{a}$ la différence des hauteurs accélératrices, aprés ouverture.

On obtient facilement

$$
\left\{\begin{array}{l}
\mathrm{H}_{a}=\mathrm{Z}+p \cdot \mathrm{Q}_{0}^{2}+\mathrm{Q}^{a} \\
\Delta \mathrm{H}_{a}=\mathrm{Q}_{1}^{2}+2 \mathrm{Q}_{0} \mathrm{Q}^{2}
\end{array}\right.
$$

$r, p=$ coefficient des pertes de charge de l'étranglement de la galerie,

$R, P=$ pertes de charge dans l'etranglement et la galerie, à pleine charge.

En reportant dans l'équation (1), on trouve finalement :

$$
\frac{Q_{0} Q_{1}}{Q_{m^{2}}{ }^{2}}+\frac{Q_{0}^{2}}{Q_{m}^{2}}=\frac{Z}{R}+\frac{\mathrm{P}}{R} \frac{Q_{0}{ }^{2}}{Q_{m^{2}}}
$$

En posant :

$$
\frac{Q_{0}}{Q_{m}}=q_{0} \quad \frac{Q_{1}}{Q_{m}}=q_{1} \quad \frac{Z}{\mathrm{P}}=z \quad \frac{\mathrm{R}}{\mathrm{P}}=\gamma
$$

on peut écrire:

$$
z=\gamma q_{0} q_{1}-q_{0}^{2}(1-\gamma)
$$

La courbe représentative de (3) peut che tracée dans le plan $(Z, Q)$. Si on se donne par exemple $Q_{1}$, l'intersec-

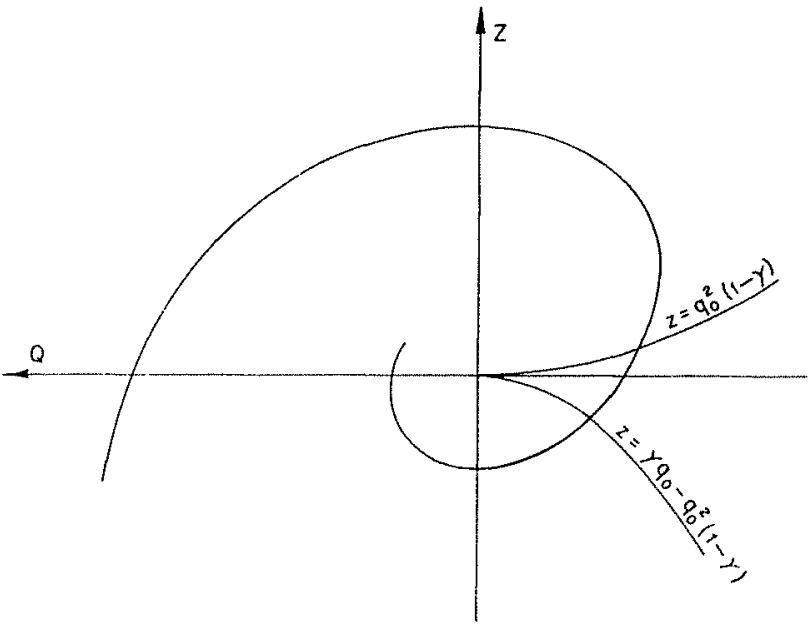

Ii1.. 2 
tion de la courbe (3) avec la courbe de fermeture donne le point le plus défavorable, pour la réouverture $Q_{1}$ donnée.

La courbe (3) est représentée par la somme de la parabole fixe, indépendante de $\varphi_{0} z=q_{0}{ }^{2}(1-\gamma)$ et de la droite $: z=\gamma q_{0} q_{1}$ à coefficient angulaire variable, dépendant de $q_{1}$. Si on fait varier $q_{1}$ entre ses limites 0 et 1 , on peut délimiter sur la courbe de fermeture la zore des points les plus défavorables.

Si, en particulier, on fait $R=0$, l'équation (3) r'eprésente l'équation de la parabole des pertes de charges dans la galerie :

$$
Z+\mathrm{P}\left(\frac{\mathrm{Q}_{0}}{Q_{m}}\right)^{z}=0
$$

Dans ce cas, quel que soit $q_{2}$ (degré d'ouverture instantané final), le point défavorable est situé à l'intersection de la courbe représentant les pertes de charge dans la galerie et la courbe représentant le mouvement de ferineture.

\section{Bouvard et J. Molisert.}

M. Escande remarque que les abaques qu'il a proposés donnent des résultats directement utilisables poar des chambres à section constante, alors que le cas traité par M. Bovvard correspond à une chambre avec épanonissements supérieurs.

M. Bouvard précise que la cheminee, à propos de laquelle ces études ont été établies, est à épanouissements successifs et comporte un étranglement dont la perte de charge est de' 16 mètres dans le sens de la montée et de l'ordre de 32 mètres dans le sens de la descente pour le débit maximum; mais, dans le cas de manceuvres de fermeture et d'ouverture successives, le débit à travers l'étranglement lié à la manœuvre d'ouverture, peut s'ajouter au débit inverse dans la galerie (et par suite dans l'étranglement) provenant de l'oscillation résiduelle de la fermeture. La perte de charge à travers l'étranglement peut donc atteindre une valeur beaucoup plus forte, qui pourrait assez facilement entraîner des phénomènes de cavitation (pouvant s'avèrer redoutables) dans la galerie, sous l'étranglement. Par ailleurs, M. Bouvand précise que la façon dont $M$. Molbert et lui-même ont ètudiè le problème des manœuyres successives (spécialement la formule donnant le point le plus défavorable) s'applique à une cheminée de forme quelconque à étranglement optimum ou non, ou même sans étranglement.

M. Chamayou estime que dans les ouvrages qui ne sont pas conditionnés pour les circonstances les plus défavorables au moment de la construction, l'utilisation des abaques de M. Escande, d'ailleurs très intéressantes pour satisfaire l'intuition des électriciens qui assimilent le phénomène à la fermeture ou à l'ouverture d'un disjoncteur sur un point quelconque de la sinusoïde, ne peut consister qu'à donner des consignes d'exploitation : or, des consignes seraient difficiles à concevoir parce qu'elles seraient basées, non sur la hauteur, mais sur la vitesse ascensionnelle de l'eau dans la cheminée d'équilibre.

M. Escande pense que cette consigne pourrait ne pas être trop serrée et consister, à la suite d'un déclenchement total, à imposer un délai de réenclenchement ou à réduire la course des régulateurs de manière à éviter toute oscillation dangereuse.

Se référant à un calcul analogue effectué par E.D.F. pour la cheminée d'équilibre de Montpezat, M. Remenie. Ras indique que la manœuvre la plus défavorable envisagée consiste en une ouverture totale débutant après un * déclenchement » à pleime charge, à l'instant où la vitesse de l'eau dans la cheminée est maximum et dirigée vers le bas; c'est là un cas exceptionnel dont la probabilité de réalisation est faible en exploitation normale; pour l'éviter complètement, on pourrait interdire toute ouverture en bloquant le distributeur de la turbine pendant toute la période où la vitesse dans la cheminée est comprise dans un certain domaine facile à déterminer; la mesure de cette vitesse - au droit du rétrécissement par exemple - pourrait être réalisée par une tuyère électromagnétique (1).

Plus généralement, on peut avoir intérêt à limiter la durée et l'amplitude des oscillations des cheminées d'équilibre afin de réduire la fatigue des revêtements de galerie, souvent mal adaptés pour résister à de fortes fluctuations de pression; on peut concevoir un servomécanisme qui, suivant les indications de la tuyère électromagnétique ci-dessus, manouvrerait le distributeur de façon à assurer un amortissement rapide des oscillafions suivant un processus de « contre-entretien 》 exactement inverse de celui considéré dans la théorie de Thoma sur la stabilité des cheminées d'équilihre.

M. Chamayou suggère de neutraliser la baisse de pression au pied des cheminées par l'adduction dans celles-ci d'une réserve d'eau contenue dans une canalisation souterraine et qui serait làchée au moment opportum.

M. Escande ajoute, au sujet des étranglements dissymetriques qu'une solution simple consisterait à prévoir des étranglements fixes et un clapet dans l'autre sens, si l'on pouvait avoir une confiance absolue dans le fonctionnement mécanique du clapet.

M. Duranton signale que lors des mancruvres d'ouverture selon les tendances actuelles (ouvertures partielles instantanées et ouvertures totales linéaires), il est possible de renforcer l'action de l'étranglement pour les débits fournis par la chambre d'équilibre. Ceci améliore l'amortissement des oscillations en masse et réduit sensiblement certains des écarts entre mancuvre unique ef mancuvre superposée obtenus par M. Escande dans l'hypothèse de l'ètranglement symétrique optimum à la fermeture.

D'une façon générale, pour les prises de charge consécutives à des déclenchements, l'étranglement symétrique suffit à procurer un amortissement permettant le réenclenchement sans risques an bout de quelques minutes: tel a été, par exemple, le cas de Montperat.

M. Bovvard remarque que l'étranglement a des conséquences défavorables dans la mesure où il amplifie la rapidité des variations de pression dans la galerie. La pression dans la galerie est, en effet, plus grande que le niveau dans la cheminée à la montée de l'eau et au contraire plus petite à la descente. Ce phénomène peut avoir des conséquences assez défavorables sur le comportement des revêtements qui, en fait, ont à encaisser la différence de pression entre celle qui règne à l'intérieur de la galerie d'amenée, et celle qui règne dans le terrain. Si les variations de pression sont rapides, cette dernière n'a pas Ie temps de s'ajuster à la pression intérieure.

M. Duranton conclut en rappelant que l'étranglement joue dans le sens de l'atténuation de l'effet essentiel de la cheminée d'équilibre et qu'il s'agit, en l'occurrence, de rechercher un optimum.

M. le Président remercie M. Escande.

(1) G. Remenieras et Hermant : "Mesure électromagnétique des vitesses dans les liquides" (Mémoires et Travanx de la $S . H . F ., n^{\circ}$ II, 1954). 